\title{
Symptomatic Compressive Pneumocephalus: Rare Complication of Posterior Spine Surgery
}

\author{
Youssef Joulali*, Faycal Lakhdar, Mohamed Benzagmout, Khalid Chakour and Mohamed el Faiz Chaoui \\ Department of Neurosurgery, University Hospital Hassan II Fez, Morocco
}

*Corresponding author: Youssef Joulali, Department of Neurosurgery, University Hospital Hassan II Fez, Morocco

Received: 監June 23, 2020

Published: 海June 29, 2020

\begin{abstract}
Pneumocephalus is the presence of air intracranially, this pathology is mostly seen in traumatic injury, however it remained exceptional in posterior spine surgery. In this study, we report a clinical case of a 66 years old woman who was operated for lumber spinal stenosis with spondylolisthesis presented a drowsiness, CT scan showed a compressive pneumocephalus (mount fuji sign) and pneumorrachis. A conservative treatment has been set up. The evolution was marked by improving its state of consciousness, the patient was discharged on day 10 post operatively with a brain CT that showed regression of PC.
\end{abstract}

Keywords: Pneumocephalus; Spine; Surgery; Posterior; CT Scan

\section{Introduction}

Pneumocephalus (PC) is defined as the presence of air intracranially. This condition is seen frequently in the cranio-facial trauma, surgery of brain tumor or infectious lesions. The PC can be epidural, subdural, intraparenchymal or intraventricular. The PC after a spine surgery is a rare complication that must systematically lead to look for a breach of the dura mater. Most often we find a pneumorachis, which is usually asymptomatic, confirms the origin of this complication. We describe below the case of a patient who was treated in our department of Neurosurgery of CHU Hassan II of Fez who presented a PC after posterior spine surgery.

\section{Clinical Case}

Woman 66 years old, with past medical history of hypertension on treatment for 4 years. She has one-year history of back pain with intermittent claudication. On examination a lumbar spinal pain syndrome without associated neurological signs was found. The magnetic resonance imaging (MRI) objectified lumber spinal stenosis with a L4-L5 spondylolisthesis grade I (Figure 1). The patient was operated in the prone position where a L4-L5 laminectomy and foramenotomy with Harrington rod and screw fixation at L4-L5-S1 (show check lateral x-ray). However, no breach of the dura or flow of cerebrospinal fluid (CSF) had been noticed intraoperatively. The clinical evolution was marked by improved pain and claudication immediately after surgery. At day 2 postoperatively, the patient presented with drowsiness with two vomiting episodes, which justified the realization of a brain Computed Tomography (CT) scan that showed the presence of a compressive frontal PC (sign of Mount Fuji) (Figure 2), a lumbar CT spine was performed with transverse sections that showed a pneumorachis (PR) with an intra canalicular screw (Figure 3). The patient was put on rehydration and put in the Trendelenburg position. The evolution was marked by improving its state of consciousness, the patient was discharged on day 10 post operatively with a brain CT that showed regression of PC (Figure 4). 


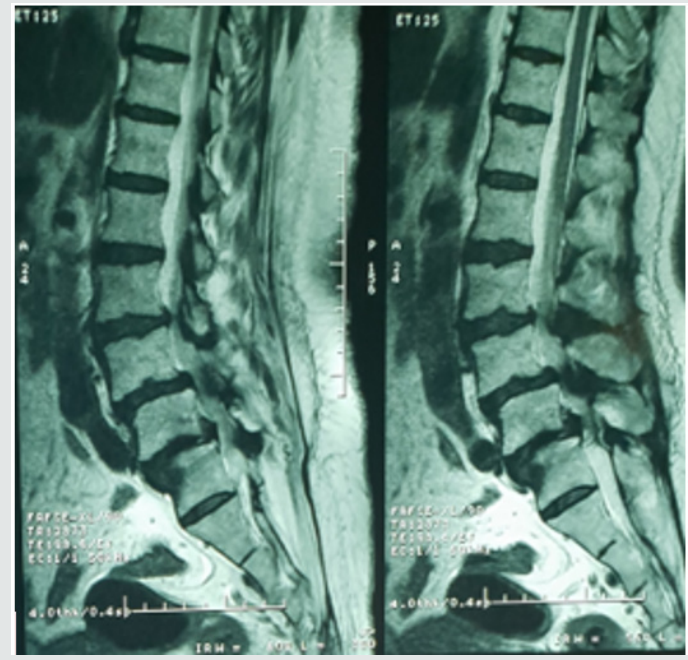

Figure 1: Preoperative MRI T2: Spinal Stenosis with Spondylolesthesis L4-L5.

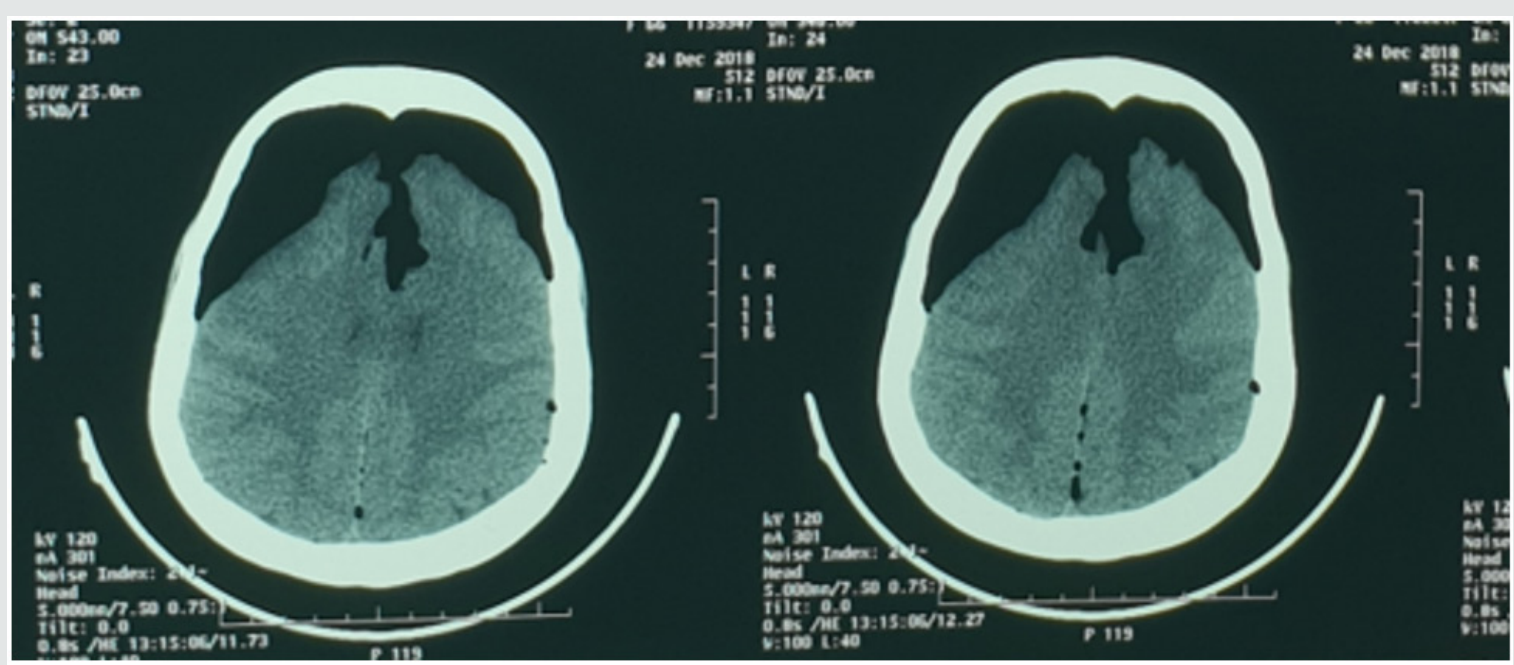

Figure 2: CT Scan shows frontal Pneumocephalus (mountfuji sign).

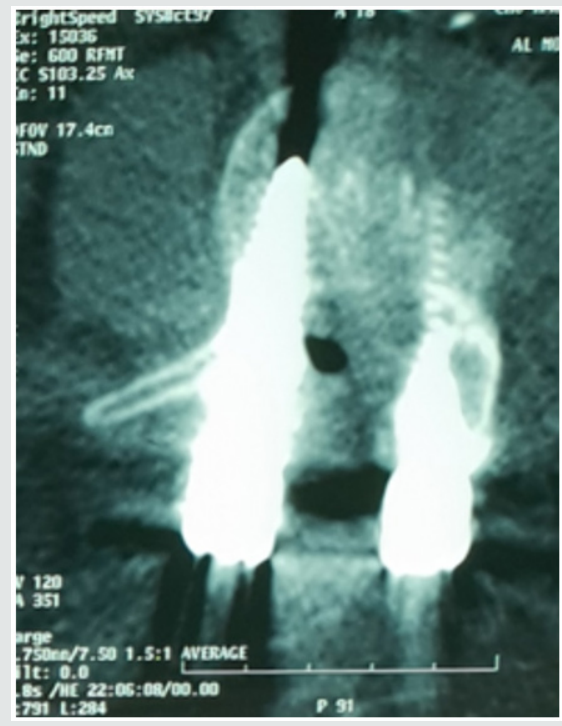

Figure 3: CT scan axial cut showing pneumorrachis. 


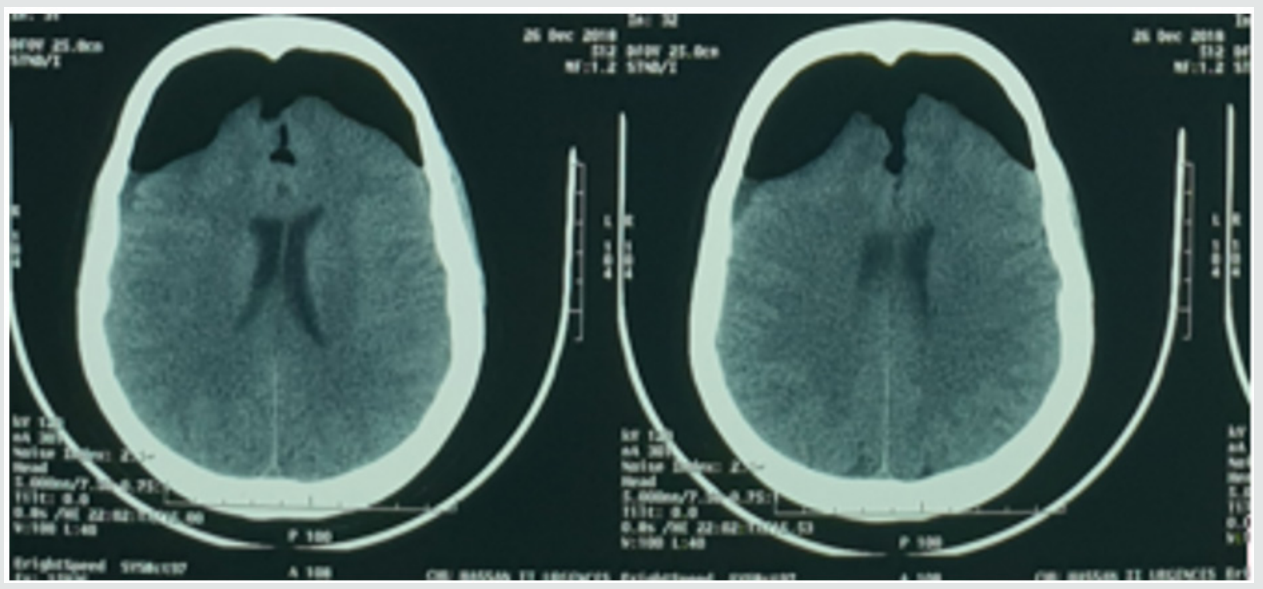

Figure 4: Control CT scan: regression of the pneumocephalus.

\section{Discussion}

Pneumocephalus (PC) is defined as the presence of air intracranially whose location can be extra-axial or intra-axial [1]. According to Markham, PC in $74 \%$ is due to a trauma, $24 \%$ to an infectious cause, and $9 \%$ following surgery of the skull [2]. The PC can also be observed following a lumbar puncture or as a rare complication of spinal anesthesia $[3,4]$. The PC is due to two mechanisms: 'the inverted soda bottle mechanism' and 'the ball valve mechanism'. The inverted soda bottle: the flow of CSF due to a breach of the dura mater, will generate negative intracranial pressure, therefore, the air will penetrate in to replace intracranial CSF to have a normal intracranial pressure. $[2,5,6]$.

The ball valve mechanism: the pressure gradient increases and will form a large air collection intracranially [5-7], this pressure gradient is formed when the pressure exceeds the pressure extracranially, and intracranial presence of a dural tear. This gradient increase will generate an increase in pressure of paranasal cavities that will push the air through intracranial dural tears $[5,6,8]$. The leaving of a redon drain predisposes the patient to a PC in the presence of a CSF leak [9]. Ayberk et al. reported a case of spontaneous PC without breach on the dura mater, they then concluded that the increase in intra-abdominal pressure due to the position is the cause of this PC [10].

In the review of the literature made by Gauthé R. et al. About spontaneous pneumocephalus after spine surgery, eight cases have been published and iatrogenic breach was noted in six cases [11]. The presentation is not specific, the PC can then be asymptomatic as it can be a varied clinical picture ranging from headache to the convulsive seizures or downright consciousness disorder, the neurosurgeon must be attentive to the signs in case of surgery spine. CT remains the gold standard for the diagnosis of PC and shows the sign of mount fuji. The management of PC remains controversial to this day there is no well-defined protocol. The bed rest, oxygen therapy and hyperhydration are usually offered. Nowak et al reported a case of PC associated with acute subdural hematoma [12]. Apart from this case, the authors instead opt for conservative treatment.

Spontaneous resorption of the PC may be seen in $85 \%$ of patients between 2-3 weeks, during which prophylactic treatment of meningitis can be considered in case of CSF leak [9].

\section{Conclusion}

The PC is a rare complication in posterior spine surgery, the clinical picture is variable and nonspecific. The realization of a brain CT scan is compulsory in every patient with neurological symptoms postoperatively. The conservative approach may be sufficient even in the presence of severe neurological signs.

\section{References}

1. Schirmer CM, Heilman CB, Bhardwaj A (2010) Pneumocephalus: case illustrations and review. Neurocrit Care 13(1): 152-158.

2. Markham JW (1967) The clinical features of pneumocephalus based upon a survey of 284 cases with report of 11 additional cases. Acta Neurochirurgica 16: 1-78.

3. Giraud P, Chauvet S (2013) Secondary Pneumocephalus After Lumbar Puncture: An Unusual Complication of Spontaneous Intracranial Hypotension? Clin Neurol Neurosurg 115(10): 2204-2206.

4. Ritesh Lamsal, Rajjeb K Mishra, Niraj Kumar (2018) Pneucephalus following lumbar spine surgery: A Rare cause of delayed emergence from Anesthesia. Journal of Neuroanaesthesiology and Critical Care 5(1): $30-32$.

5. Ihab Z (2012) Pneumocephalus After Surgical Evacuation of Chronic Subdural Hematoma: Is it a Serious Complication? Asian J Neurosurg $7(2): 66-74$.

6. Swenis S, Senthilkumaran $S$, Balamurgan N, Thirumalaikolundusubramanian P (2013) Tension Pneumocephalus: A Case Report with Review of Literature. Emerg Radiol 20(6): 573-578.

7. Dabdoub CB, Salas G, Silveira ED, Dabdoub CF (2015) Review of the management of pneumocephalus. Surg Neurol Int 6: 155.

8. Yun JH, Kim YJ, Yoo DS, Ko JH (2010) Diffuse Pneumocephalus: A Rare Complication of Spinal Surgery. J Korean Neurosurg Soc 48(3): 288-290. 
9. Turgut M, Akyûz O (2007) Symptomatic Tension Pneumocephalus: An Unusual Post-Operative Complication of Posterior Spinal Surgery. J Clinneurosci 14(7): 666-668.

10. Ayberk G, Yaman ME, Ozveren MF (2010) Symptomatic Spontaneous Pneumpcephalus After Spinal Fusion for Spondylolisthesis. J Clinneurosci 17(7): 934-936.
11. R Gauthé, Latrobe C, Damade C, Foulongne E, Roussignol X, et al. (2016) Symptomatic compressive pneumocephalus following lumbar decompression surgery. Orthop Traumatol Surg Res 102(2): 251-253.

12. Nowak R, Maliszewski M, Krawczyk L (2001) Intracranial Subdural Hematoma and Pneumocephalus After Spinal Instrumentation of Myelodysplastic Scoliosis. J Pediatr Orthop B 20(1): 41-45.

\section{(c) (i) \\ This work is licensed under Creative Commons Attribution 4.0 License}

To Submit Your Article Click Here:

Submit Article

DOI: $10.32474 / O J N B D .2020 .04 .000182$

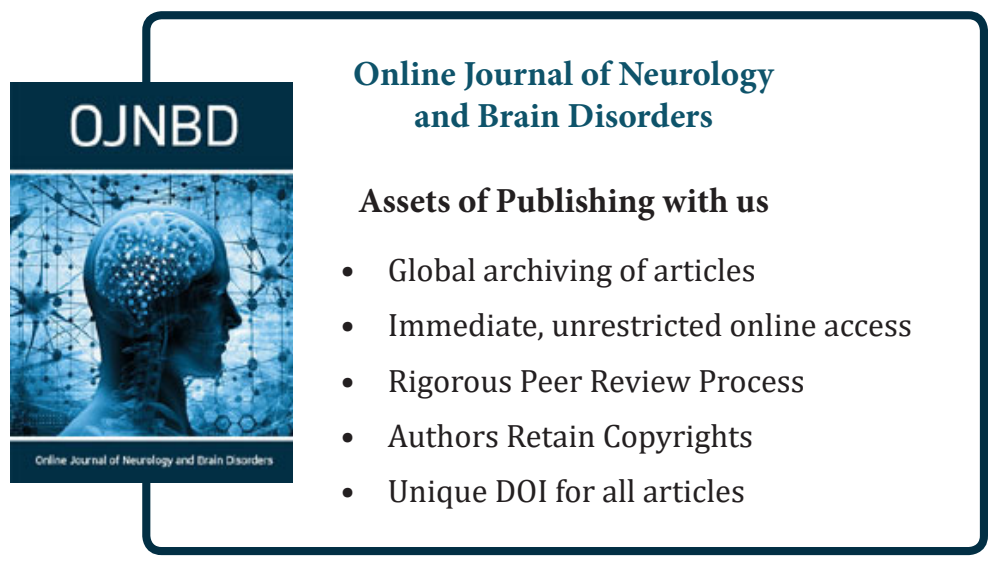

